\title{
A radical new treatment for the sick health workforce
}

\author{
Christopher B Del Mar and Naomi Dwyer
}

N o-one would deny there is a problem with the Australian health workforce. Urgent response is evident, announcements of fast moving policy decisions appearing in quick order.

But what is happening, and, more importantly, why?

The clinical disciplines emerged in the late 17th and 18th centuries as discrete crafts, often in direct competition with each other - even as late as 1880 , doctors regarded nurses as a threat to their professional sovereignty. ${ }^{1}$ Consequently, to ease potential conflict, roles and accountabilities were precisely defined. This unambiguous scope of practice was mostly effective at the time. It enabled different disciplines to get along and perform as a team in specific situations, and it promoted some - if begrudging measure of interdisciplinary respect. Acute care teams were able to undertake ward rounds, conduct operations, and care for patients successfully in hospitals. What has gone wrong since then?

\section{What are the symptoms of our clinical workforce problems?}

The ageing of the population, with increased comorbidity and the burgeoning increase in chronic disease, underscores the crisis. Diabetes will be the second most common condition affecting males by $2016 .^{2}$ We are ill-prepared for the onslaught of increasing clinical care needed by patients with chronic degenerative diseases and other demands.

Simultaneously, the supply side is declining for a variety of reasons: insufficient health students to keep pace with population growth; feminisation of the workforce; increasing medical technology sometimes, perversely, requiring more personnel; retirement patterns; ${ }^{3}$ and the changing aspirations of "Generation Y", which admires commitment to balancing lifestyle and family over duty to one's profession.

The workforce illness is neither transient nor acute, nor amenable to swift treatment - it is a serious, chronic illness. Previous failed treatments must be discarded, and we badly need a more efficient system.

\section{Provisional diagnosis}

Although the clinical landscape is changing rapidly, the primary structure of the health workforce remains the same, despite entreaty from many to rethink the traditional barriers between professions and consider developing new professions. ${ }^{4}$ There is some blurring at the margins of medical and nursing roles, but disciplinary "silos" remain entrenched, each profession defending its walls against what is seen as loss of disciplinary sovereignty. This has been a feature of the health system from early on (for example, in 1871, doctors of the failing Sydney Hospital charged the newly established nursing profession in Australia with incompetence, blaming them for the crisis; the nurses were exonerated by a New South Wales Government inquiry). ${ }^{5}$ Unease between the health professions is not yet completely resolved, and this territorialism threatens teamwork.

The contribution of older and newer disciplines is not at issue. Indeed, there is a generally favourable verdict. ${ }^{6}$ Yet, to the average

\section{ABSTRACT}

- The health workforce crisis needs radical treatment; simply educating more health workers will be insufficient, and role substitution among existing health workers is untenable.

- We propose a new class of health worker who would take on single disease or single procedure responsibilities, working mostly to protocols; and be embedded within current structures.

- We also propose modular health education which has fewer entry points into the health system, allows transfer between different disciplines, and is based on modules that can be accumulated to allow progress through the system to gain more clinical responsibility.

MJA 2006; 185: 32-34

patient, some of the professional fragmentation is arcane and subtle far beyond intuition. Few lay people can easily explain the difference in contributions between chiropractor and osteopath, or physiotherapist and occupational therapist. Rather, multidisciplinary teamwork is more important than ever, and no one clinical discipline, let alone one clinician, can ever over a short time hope to look after all the complexities of a single patient's needs, except for a very narrow range of problems.

Teamwork, the notion of fragments of care coming together as a seamless service, is particularly important for chronic care, which becomes transformed into a protocol-driven set of interdependent tasks. But this is not so easy. Effective teams now require the special contributions of so many health workers that they are burdensomely large. Not even modern information and communication technology, in the innovations tested, appears able to bring them together elegantly. Our teams are so large they are effectively unworkable unless coordinated by a powerful case manager.

Pity the person with diabetes, who confronts a logistical hurdle in setting out to access each professional necessary for his or her health care (Box): multiple appointments, travel, time and organisation. Health managers, too, face serious challenges in providing the necessary fragments in one time and place, avoiding missing any fragments and ensuring adequate interprofessional communication.

Conventional conservative managements have all failed. ${ }^{7} \mathrm{~A}$ new approach is indicated, pragmatic in application and systemic in reach.

\section{Treatment options}

The current approach is simply to demand more: more doctors, more nurses, and more allied health workers. By itself, this strategy cannot succeed. ${ }^{8}$ In the short term, we cannot train enough doctors, particularly general practitioners, let alone nurses. ${ }^{9}$ The geometrically expanding health needs of our growing and ageing community are outstripping the arithmetically increasing production of health professionals.

We need fundamental workforce innovation, changes to scope of practice, and job redesign (all properly evaluated). ${ }^{10}$ Role 


\section{Access to the health system for a person with diabetes: current $(A)$ and proposed (B) systems}

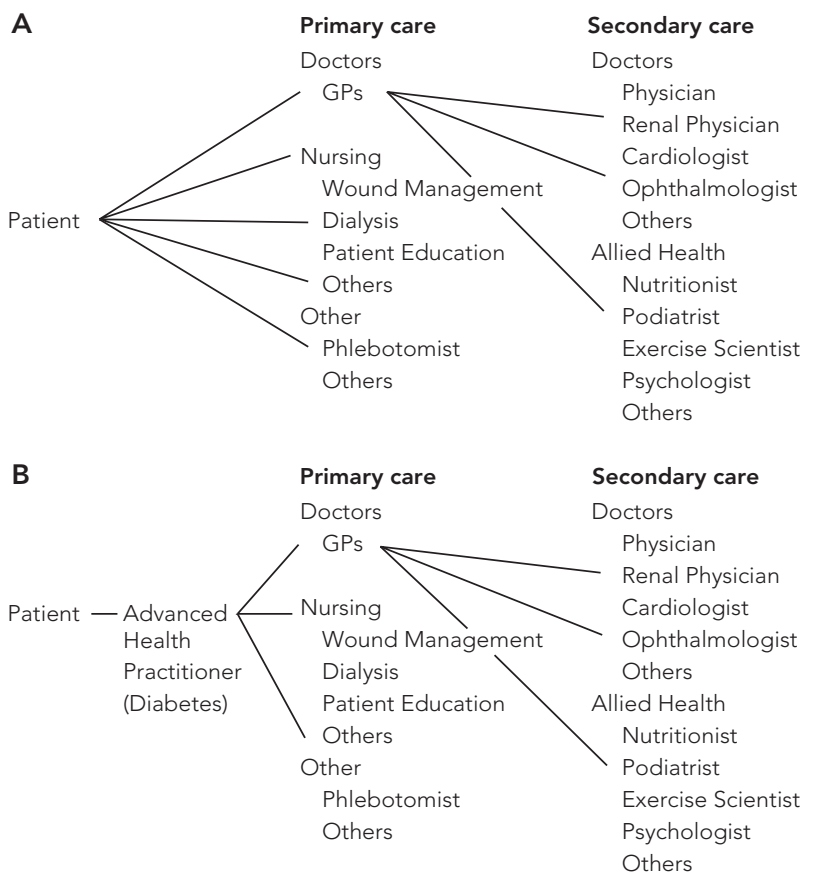

A: In the current system, the patient has to identify and make appointments with all the different health professionals necessary for his or her care. B: In our proposed system, the patient has one point of access to the health system. The Advanced Health Practitioner (Diabetes) has the clinical knowledge required for routine management of the patient (podiatry, exercise science, pharmacology, optometry, wound management, health education, social work, nutrition - and perhaps physiotherapy, occupational therapy, sexual health, etc — with communication skills, IT, evidence-based medicine, and ethics).

substitution is one approach that has been implemented, usually involving nurses replacing doctors after extra training. Examples include caring for patients with heart failure in the home (where nurses obtain better results, interestingly). ${ }^{11}$ Nurses are also specially trained for new technology roles, such as managing complex operating theatre equipment, and providing the special skills of intensive care. But requiring a complete nursing education as a prerequisite seems hopelessly inefficient if the basic skills are under-used in the new role. Moreover, $78 \%$ of the nation's nursing workforce will need to be replaced between 2003 and 2012, and there is a projected shortfall of about 6100 nurses annually. ${ }^{12}$ Nursing as a career seems to have difficulties in recruitment, presumably an image problem, that dwarf any other health profession's workforce problems. ${ }^{13}$ Recruiting from the nursing pool will only shift the problem there, creating more problems than it solves.

\section{A radical treatment plan}

We propose a new workforce rather like physician assistants of the United States. ${ }^{14}$ Our current educational machinery increasingly deepens specialisation, preparing clinicians for functions "just in case" (an emphasis on specialised functions ready to hand), rather than "just in time" (accessing such functions as needed). ${ }^{15}$ In other words, clinical work demands easy patient access to a wide range of clinical skills, yet our workforce becomes increasingly fragmented. Most clinical work for patients with multiple comorbidities increasingly requires knowledge and skills that are "mile wide" and "inch deep".

It will be important to establish a career pathway enabling new practitioners to assume new responsibilities. Ideally, this would enable them to move into the traditional roles - becoming doctors or allied health professionals.

If this sounds like science fiction, look at the experiments at the University of Southampton Hospital Trust, in the United Kingdom, where an "educational escalator" (a series of common educational modules provided by both health and educational sectors) now exists. With some modification, this could enable health workers to move either vertically or laterally into other health professions. Further, a patchwork of modules could be grouped together in different ways to form several complete sets of different professional skills, ${ }^{16}$ some common to many roles, some to all. The matrix enables health professionals to move across the tapestry in any direction needed by the health service, and preferred by the individual, dependent upon aspiration and ability. This contrasts with the current system, which looks more like Snakes and Ladders (without the ladders): to move into a new traditional discipline (say, to move from physiotherapy to occupational therapy, or even medicine) means abandoning almost everything so far achieved (prior learning being almost never recognised) to start from a bottom square again.

Most health professionals have had no experience in another health role. This makes it hard for them to recognise the challenges and pressures of each others' roles, and must ultimately engender an "us" and "them" culture. Enlarging on a new skills escalator approach could change all that.

All this raises the question of what traditional disciplines we need to retain. Do we need doctors with all their specialist divisions? The answer depends on what society wants. If it is one individual responsible for the delivery of care at separate points of the care continuum, then one person has to assume that responsibility. In the current system, that is ultimately a doctor. Certainly that responsibility is sometimes delegated to another (for example, a nurse or allied health professional, or in our new model, a new health professional: Box), and with that delegation (assuming it is done properly) goes also responsibility. But the person making the delegation is ultimately a doctor.

This solution creates a new environment in which people can enter medicine by a route different to the current one (rather like officer selection for the military, which allows "elevation through the ranks"). Everyone then would have a common entrance into health. Progress would be by merit and ability. People could advance their careers or retreat them as suits their career, personal aspirations and abilities. They would have a better understanding of each others' roles, reducing the perception of arrogance levelled at doctors. ${ }^{17}$

This would also be a good time to correct that absurd inversion of health learning: the primacy of basic science as a necessary "foundation" for clinicians, existing in slightly different forms for every health care worker. It is rather like teaching a child the principles of the gyro, the atomic structure of metal, and Boyle's Law for pressure of gases as prerequisites for learning to ride a bicycle. Basic science learning is necessary, of course, but surely at the end stage? Far more necessary is the acquisition of basic management-of-people skills, communication skills, and the abil- 
ity to comfort, which all health workers need, and which (consistently) society says is missing. ${ }^{17}$ The consequence is that all health workers should be grounded in this common core as the basic foundation, and this might include everyone in the health care sector - management, administration, reception and patient services staff. As people progress in their learning, so they drill down deeper into the basic sciences.

Our model would have people entering at several levels dependent on entry preferences, but learning the same basic modules of communication skills, an understanding of the health care system, and basic administration skills, before moving towards their preferred area of health. Because these would be common to all, moving from one role to another according to need and personal preference should become much easier.

\section{What next?}

Restructuring the whole health workforce is clearly an unimaginably difficult task. Even an interim state - with a new modular educated "Advanced Health Professionals" class coming in and shouldering some of the burden - would be difficult to achieve. There would be issues of safety and quality to overcome, and then professional licensing and registration. In addition, the interim period would require close cooperation between employer (public area health service or private groups) and educational institution. Whatever model we decide on, we need to get on with trying and testing it without delay.

\section{Competing interests}

None identified.

\section{Author details}

Christopher B Del Mar, DRCOG, FRACGP, FAFPHM, Dean, Faculty of Health Sciences and Medicine

Naomi Dwyer, BBus, Executive Officer

Bond University, Gold Coast, QLD.

Correspondence: cdelmar@bond.edu.au

\section{References}

1 Black N. Rise and demise of the hospital: a reappraisal of nursing. BMJ 2005; 331: 1394-1396.
2 Cameron AJ, Welborn TA, Zimmet PZ, et al. Overweight and obesity in Australia: the 1999-2000 Australian Diabetes, Obesity and Lifestyle Study (AusDiab). Med J Aust 2003; 178: 427-432.

3 Schofield DJ, Beard JR. Baby boomer doctors and nurses: demographic change and transitions to retirement. Med J Aust 2005; 183: 80-83.

4 Queensland Health. Smart state: health 2020. A vision for the future. Summary discussion paper. Brisbane: Queensland Health, 2002. Available at: http://www.health.qld.gov.au/health2020/ (accessed May 2006).

5 Barrett NR. The contribution of Australians to medical knowledge. Med Hist 1967; 11: 321-333.

6 Australian Institute of Health and Welfare. Australia's health 2004. Canberra: AlHW, 2004. (AlHW Catalogue No. AUS44.) Available at: http:// www.aihw.gov.au/publications/index.cfm/title/10014 (accessed May 2006).

7 Duckett SJ. Interventions to facilitate health workforce restructure. Aust New Zealand Health Policy 2005; 2: 14.

8 Forster P (Chair). Queensland health systems review. Final report. Brisbane: Queensland Health, 2005. Available at: http:// www.health.qld.gov.au/health_sys_review/final/default.asp (accessed May 2006)

9 Joyce CM, McNeil JJ, Stoelwinder JU. More doctors, but not enough: Australian medical workforce supply 2001-2012. Med J Aust 2006; 184: 441-446.

10 Australian Government Productivity Commission. Australia's health workforce. Position paper. Melbourne: Productivity Commission, 2005.

11 Stewart S, Marley JE, Horowitz JD. Effects of a multidisciplinary, homebased intervention on unplanned readmissions and survival among patients with chronic congestive heart failure: a randomised controlled study. Lancet 1999; 354: 1077-1083.

12 Australian Health Workforce Advisory Committee. The Australian nursing workforce - an overview of workforce planning 2001-2004. AHWAC report 2004.2. Sydney: AHWAC, 2004. Available at: http:// www.health.nsw.gov.au/amwac/reports.html (accessed May 2006).

13 Buerhaus PI, Staiger DO, Auerbach DI. Implications of an aging registered nurse workforce. JAMA 2000; 283: 2948-2954.

14 Physician assistant and nurse practitioner workforce trends. Am Fam Physician 2005; 72: 1176.

15 Duckett S. Health workforce design for the 21st century. Aust Health Rev 2005; 29: 201-210.

16 Humphris D, Hean S. Educating the future workforce: building the evidence about interprofessional learning. J Health Serv Res Policy 2004; 9 (Suppl 1): 24-27.

17 National Health and Medical Research Council. Communicating with patients: advice for medical practitioners. Canberra: NHMRC, 2004. Available at: http://www.nhmrc.gov.au/publications/synopses/ e58syn.htm (accessed May 2006).

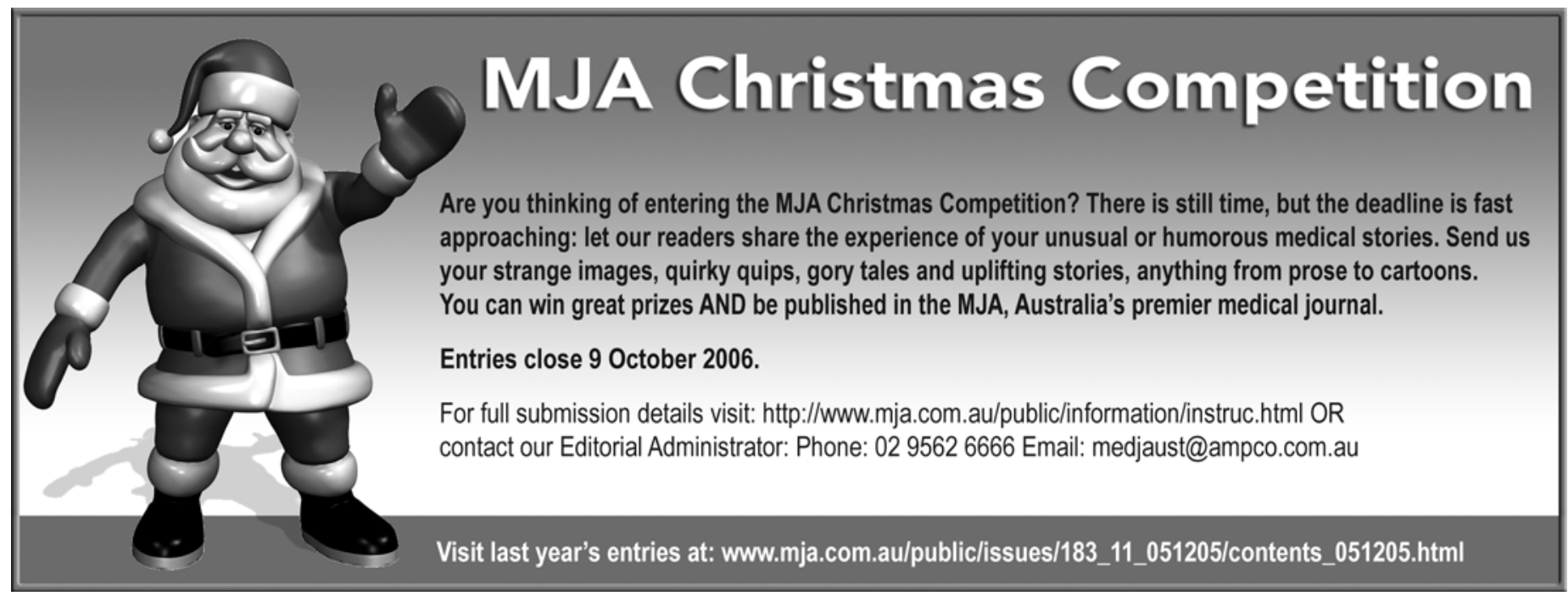

